\title{
Reducing social inequalities in the oral health of an adult population
}

\author{
Manoelito Ferreira SILVA \\ JUNIOR(a) iD \\ Maria da Luz Rosário de \\ SOUSA $^{(b)}$ iD \\ Marília Jesus BATISTA ${ }^{(c)}$ iD \\ (a) Universidade Estadual de Ponta Grossa \\ - UEPG, Department of Dentistry, Ponta \\ Grossa, PR, Brazil. \\ (b) Universidade Estadual de Campinas - \\ Unicamp, School of Dentistry, Department \\ of Health Sciences and Pediatric Dentistry, \\ Piracicaba, SP Brazil. \\ (c) Universidade Estadual de Campinas - \\ Unicamp, School of Medicine, Department \\ of Community Health, Jundiaí, SP, Brazil.
}

Declaration of Interests: The authors certify that they have no commercial or associative interest that represents a conflict of interest in connection with the manuscript.

\section{Corresponding Author:}

Marília Jesus Batista

E-mail:mariliajbatista@yahoo.com.br

Submitted: April 8, 2019

Accepted for publication: September 1, 2019

Last revision: November 19, 2019
Abstract: The objective of this study was to evaluate the association between social inequality indicators and oral health conditions in an adult population. This prospective cohort study assessed a probabilistic sampling of adults (aged 20-64 years) living in Piracicaba, São Paulo, Brazil. Oral examinations were performed in 2011 and 2015, conducted at home, and used the decay-missing-filled (DMFT) index of permanent teeth, the Community Periodontal Index (CPI), and the visible biofilm criterion. A questionnaire was administered to determine demographic and socioeconomic aspects and dental services used, and collect oral health-related quality of life (OHRQoL) data. Social inequality indicators were evaluated according to social class (high, middle or low) and type of dental service used (public, health insurance or private), and compared with oral health conditions (visible biofilm, DMFT and incidence of tooth loss, periodontal pockets and bleeding, and OHRQoL), evaluated between 2011 and 2015. Analysis using chi-squared or Fisher tests $(p<0.05)$ and Cochran's $Q$ test was conducted separately for each category analyzed between 2011 and 2015 ( $\mathrm{p}$ < 0.05). A total of 143 adults who participated in an earlier study were examined after four years of follow-up. Although the occurrence of oral disease did not decrease over the study period (4 years), there was a reduction in inequality among lower social classes in regard to presence of tooth decay and oral health impact on self-perceived quality of life between 2011 and 2015 ( $p<0.05$ ). These results suggest that the Brazilian National Oral Health Policy has achieved its principles, especially that of greater equity.

Keywords: Health Status Disparities; Health Services Research; Quality of Life; Oral Health; Epidemiology.

\section{Introduction}

The Social Determinants of Health (SDH) involve social, economic, cultural, ethnic/racial, psychological and behavioral aspects, ${ }^{1}$ and comprise factors such as, income, work, education, housing, transport, culture, leisure, difficult access to health services, sustainable environment and social support networks. These factors may lead to injustices in health, ${ }^{2}$ and are social inequality markers in the distribution and occurrence of oral diseases. 
Oral health is a strong socioeconomic and behavioral marker ${ }^{3}$ that can be used to study health inequalities in regard to oral diseases. Although oral diseases are preventable, they are highly prevalent in the world population. Untreated caries in permanent teeth is the most prevalent, ${ }^{4}$ and severe periodontitis is the sixth most prevalent condition in the world. ${ }^{5}$ When left untreated, both have tooth loss as a final outcome. ${ }^{6,7}$ These three oral health conditions (caries, periodontitis and tooth loss) have affected the health of the world's population the most in the last two decades. ${ }^{8}$ There has been no reduction in untreated caries in adults in the last decades, ${ }^{4,5}$ and it is still considered a public health problem.

The general health situation of a country not only reflects its total wealth, but also depends on how its economy is distributed among the population. ${ }^{9}$ Brazil is a country marked by inequal distribution of wealth. ${ }^{9}$ In 1988, a Brazilian Unified Health System (SUS) was proposed, with guiding principles of universal, equalitarian and comprehensive healthcare services. However, oral health was not a priority for SUS at the beginning, and remained one of the main obstacles to meeting the healthcare needs of the Brazilian population, ${ }^{10}$ where the poor were eight times more likely to never have visited a dentist. ${ }^{11}$ In this respect, the "Smiling Brazil" National Oral Health Policy was implemented in 2004, and is the largest public oral healthcare program in the world, responsible for expanding oral health coverage for primary healthcare, and providing specialized oral health services such as periodontics, minor oral surgeries, oral diagnoses, prostheses and special attention to patients with disabilities ${ }^{10,11}$. Although this program enabled greater access to and use of dental healthcare services in Brazil, it is uncertain whether the inequalities concerning these services persist even after access was improved. ${ }^{12}$ Thus, a model was sought to demonstrate the consistency of results of a public oral healthcare service that has reduced the occurrence of oral disease with greater equity.

Studies on inequalities have increased, especially in developed countries, ${ }^{13,14}$ however, most of them are cross-sectional analyse ${ }^{14,15,16,17}$ of young people, ${ }^{13,16}$ adults $^{14,15,16}$ or older adults, ${ }^{14,17}$ or else assess certain oral health conditions, such as healthcare service use and health behaviors, ${ }^{13,14,18}$ as well as some clinical conditions, like dental caries. ${ }^{16}$ A cohort study of an adult population is a relevant tool that can be used to identify what oral conditions may be more susceptible to social inequalities. The objective of this study was to evaluate the association between social inequality indicators and oral health conditions in an adult population after a four-year period.

\section{Methodology}

\section{Design and ethical aspects}

This study was part of a research project entitled Prospective Cohort Study of Adult Oral Health in Piracicaba, SP, Brazil ${ }^{19}$ and was approved by the Research Ethics Committee of the Piracicaba Dental School, University of Campinas (number 177/2009).

\section{Sample}

The first study was conducted between June and September 2011, and the sample had 248 adults aged between 20-64 years, and living in Piracicaba ${ }^{19}$. A new examination was held between the same months of 2015.

The exclusion criteria of the study were volunteers who had physical and psychological states that prevented them from understanding certain issues and future clinical procedures, individuals not found in the selected household during the data collection period, or those who refused to participate in the study. In an effort to ensure a smaller loss to follow-up, census tracts drawn at baseline were not considered an inclusion criterion in the follow-up period.

At the baseline, 248 adults participated in the study, totaling the minimum number required for a representative sample of adults from the city. ${ }^{19}$ However, after four years of follow-up, the sample consisted of 143 examined individuals, comprising the final sample used in this study.

\section{Data collection}

The study comprised a clinical oral examination and an interview. ${ }^{19}$ The examination assessed pit-and-fissure caries using the Decayed, Missing and Filled Teeth (DMFT) index of permanent teeth, 
the Community Periodontal Index (CPI) and the visible biofilm criterion. Collection was carried out in households, under natural lighting (without prophylaxis or drying), using CPI probes and plain mouth mirrors, as recommended by the World Health Organization.

At baseline, the initial collection (2011) was performed by one rater, who was trained using theoretical and practical sessions lasting a total of 20 hours, and who obtained at least $90.0 \%$ reliability for the evaluation of pit-and-fissure caries and periodontal condition. Intrarater reliability ranged from $96.5 \%$ to $100.0 \%$, which is within the reliability standards. The Kappa index ranged from 0.89 to 1.00 for all clinical conditions, and was also within the reliability standards. ${ }^{19}$ At follow-up, two raters participated in the data collection, and the rater for the baseline period was the "gold standard" for calibration. The raters were trained to evaluate caries, periodontal condition and visible biofilm. There were theoretical and practical discussions totaling 20 hours before obtaining intrarater reliability above $96.5 \%$, and interrater reliability above $90.0 \%$. The Kappa index ranged from 0.89 to 1.00 for all clinical conditions, and was within the reliability standards. ${ }^{19}$ In addition, each volunteer answered a questionnaire on demographic (sex, age, race and marital status) and socioeconomic factors (personal and family income, educational level, occupation, type of residence and number of household residents), use of dental services and oral health impact on self-perceived quality of life, ${ }^{20}$ in both study phases.

\section{Variables}

The dependent variables were social inequality markers, based on the following criteria:

a. Social class: the socioeconomic classification was carried out according to Graciano et al., ${ }^{20}$ using a score based on educational level, household income, occupation, type of residence and number of household residents, and resulted in six social classes. The social classes of this study were grouped into the categories of high, medium and low.

b. Type of dental service used: last dental visit, and public, private and health insurance.
The independent variables were oral health conditions, observed in the clinical exam, as follows: a. Visible biofilm: considered 'yes' (at least one tooth surface with visible dental biofilm), or 'no' (all tooth surfaces without visible dental biofilm);

b. Decayed teeth (D component of the DMFT): considered 'yes' (or present) (at least one tooth decay), or 'no' (absence of tooth decay);

c. Filled teeth (F component of the DMFT): considered 'yes' (at least one filled tooth), or 'no' (absence of filled teeth);

d. Missing teeth (M component of the DMFT): considered 'yes' (at least one missing tooth) (Codes 4 or 5 of the index), or 'no' (absence of missing teeth);

e. Bleeding gums: measured using the Community Periodontal Index (CPI), and considered 'yes' if at least one sextant presented bleeding (Code 1 of the index);

f. Periodontal disease: measured using the CPI, and considered 'yes' if at least one sextant presented clinical attachment loss (CAL) of more than $4 \mathrm{~mm}$ (Code 3 and 4 of the index);

g. Impact on Oral Health-Related Quality of Life (OHRQoL) was measured by the Oral Health Impact Profile-14 (OHIP-14) ${ }^{21}$ prevalence, according to the proportion of individuals whose response to at least one of the 14 items was "fairly often/very often."

\section{Data analysis}

The data were tabulated using the Statistical Package for the Social Sciences, version 20.0. A descriptive analysis was performed to obtain the absolute and percentage distribution of the variables under study. Statistical analyses were also performed between each variable of the social inequality markers and the oral health conditions, in 2011 and 2015, separately, by the chi-squared or Fisher tests $(\mathrm{p}<0.05)$. In addition, the results for 2011 were compared with those of 2015, using Cochran's Q test $(\mathrm{p}<0.05)$. Equiplots (www.equidade.org/equiplot) were performed with Stata 12.1 (StataCorp LP, College Station, USA), and used for the graphic display of inequalities between 2011 and 2015. 


\section{Results}

Most of the sample was composed of older women in a de facto relationship, with a family income up to two minimum monthly wages, educational level of over 11 years, and belonging to the middle class (Table 1).

Regarding the study population, 54 (39.1\%) volunteers presented visible biofilm in 2011, and 53 (38.4\%) in 2015. A total of 47 (32.9\%) and 49 (34.3\%) had dental caries in 2011 and 2015, respectively. Twenty $(14.0 \%)$ versus eighteen $(12.6 \%)$ individuals did not have a restoration or tooth loss in 2011 compared with 2015, respectively. A periodontal pocket above

Table 1. Demographic and socioeconomic characteristics at baseline and follow-up of adults living in Piracicaba, SP, Brazil, 2011-2015.

\begin{tabular}{|c|c|c|}
\hline \multirow{2}{*}{ Characteristic* } & Baseline & Follow-up \\
\hline & 2011 n (\%) & 2015 n (\%) \\
\hline \multicolumn{3}{|l|}{ Demographic } \\
\hline \multicolumn{3}{|l|}{ Sex } \\
\hline Man & $40(28.0)$ & $40(28.0)$ \\
\hline Woman & $103(72.0)$ & $103(72.0)$ \\
\hline \multicolumn{3}{|l|}{$\operatorname{Age}^{* *}$} \\
\hline 20-44 years-old & $70(49.0)$ & $70(49.0)$ \\
\hline 45-64 years-old & $73(51.0)$ & $73(51.0)$ \\
\hline \multicolumn{3}{|l|}{ Marital status } \\
\hline Stable union & $112(78.3)$ & $108(75.5)$ \\
\hline No stable union & $31(21.7)$ & $35(24.5)$ \\
\hline \multicolumn{3}{|l|}{ Socio-economic } \\
\hline \multicolumn{3}{|l|}{ Household income } \\
\hline$\leq 1$ minimum wage & $07(4.9)$ & 09 (6.3) \\
\hline $1-2$ minimum wages & $16(11.2)$ & $18(12.6)$ \\
\hline$\geq 2$ minimum wages & $116(81.1)$ & $94(65.7)$ \\
\hline Not informed & $04(2.8)$ & $22(15.4)$ \\
\hline \multicolumn{3}{|l|}{ Education level } \\
\hline$\leq 4$ years & $30(21.0)$ & $31(21.7)$ \\
\hline $5-10$ years & 37 (25.9) & $34(23.8)$ \\
\hline$\geq 11$ years & $76(51.3)$ & $78(54.5)$ \\
\hline \multicolumn{3}{|l|}{ Social class } \\
\hline Low & $23(16.1)$ & 17 (1 1.9) \\
\hline Medium & $96(67.1)$ & $71(49.6)$ \\
\hline High & $24(16.8)$ & $18(12.6)$ \\
\hline Data missing & - & $37(25.9)$ \\
\hline
\end{tabular}

*Data referring to the collection held in 2011 and 2015; ** Data referring to the collection held in 2011.
$4 \mathrm{~mm}$ was present in at least one sextant in $43(30.1 \%)$ and $50(35.0 \%)$ adults assessed, and 71 (49.6\%) and $58(40.6 \%)$ individuals reported at least one severe impact of oral health on self-perceived quality of life (Table 2 and 3).

At the baseline, tooth decay ranged according to social class gradients (Figure 1 ) and type of service used ( $\mathrm{p}<0.05)$ (Tables 2, 3 and 4) (Figure 2). The social class showed an association with visible biofilm and with periodontal pockets over $4 \mathrm{~mm}$ (2011 and 2015) (Table 2), and between type of service used for biofilm (2015) and oral health impact on self-perceived quality of life (2011) (Table 3) (Figure 2). A reduction in inequality was observed among the lower social classes regarding tooth decay and oral health impact on self-perceived quality of life between 2011 and 2015 (Table 2).

\section{Discussion}

Associations were observed between inequalities and oral health conditions of the adults in the study. The lowest social class stratum and the public service users showed an association between presence of biofilm and periodontal pockets after the four years of the study. However, a reduction in the impact of social inequalities was found among lower social classes, regarding tooth decay and oral health impact on self-perceived quality of life. This is because of an association observed in 2011 but not in 2015, and the great difference after the four years of follow-up. This result was different from another Brazilian study that established increased access in these four years, but no reduction in socioeconomic inequalities regarding the use of dental services between 2003 and 2008. ${ }^{12}$ This longitudinal study was conducted among the same individuals as our study, and used an expanded adult age group. Its results are of great relevance to dentistry, because they infer an oral disease distribution that can help us understand the impact of public policies throughout the years.

Untreated early-stage caries and periodontal disease have tooth loss as the main consequence. ${ }^{7}$.It is difficult to think about reducing tooth loss without reducing its main clinical risk factors: caries and periodontal disease. ${ }^{7}$ However, despite advances in 
Table 2. Association between social class and adult oral health variables. Piracicaba, SP, Brazil, 201 1-2015.

\begin{tabular}{|c|c|c|c|c|c|c|c|c|c|c|}
\hline \multirow{4}{*}{ Variables } & \multicolumn{10}{|c|}{ Social class } \\
\hline & \multicolumn{5}{|c|}{ Baseline* } & \multicolumn{5}{|c|}{ Follow-up* } \\
\hline & High & Medium & Low & Total & \multirow{2}{*}{$p$-value } & High & Medium & Low & Total & \multirow{2}{*}{$p$-value } \\
\hline & n (\%) & n (\%) & n (\%) & n (\%) & & n (\%) & n (\%) & n (\%) & n (\%) & \\
\hline \multicolumn{11}{|c|}{ Visible biofilm } \\
\hline No & $19(22.6)$ & $56(66.7)$ & $9(10.7)$ & $84(100.0)$ & 0.019 & $21(24.1)$ & $56(64.4)$ & 10 (1 1.5) & 87 (100.0) & 0.006 \\
\hline Yes & $5(9.3)$ & $35(64.8)$ & 14 (25.9) & $54(100.0)$ & & $3(5.7)$ & $37(69.8)$ & $13(24.5)$ & 53 (100.0) & \\
\hline \multicolumn{11}{|c|}{ Decayed teeth } \\
\hline No & $23(24.0)$ & $63(65.6)$ & $10(10.4)$ & $96(100.0)$ & 0.001 & 19 (20.2) & $63(67.0)$ & 12 (12.8) & $94(100.0)$ & 0.150 \\
\hline Yes & $1(2.1)$ & $33(70.2)$ & $13(27.7)$ & 47 (100.0) & & $5(10.2)$ & 33 (67.3) & 11 (22.5) & 49 (100.0) & \\
\hline \multicolumn{11}{|l|}{ Filled teeth } \\
\hline No & $2(10.0)$ & $14(70.0)$ & $4(20.0)$ & $20(100.0)$ & 0.642 & $2(11.1)$ & 14 (77.8) & $2(11.1)$ & $18(100.0)$ & 0.589 \\
\hline Yes & 22 (17.9) & $82(66.7)$ & $19(15.4)$ & 123 (100.0) & & $22(17.6)$ & $82(65.6)$ & $21(16.8)$ & 125 (100.0) & \\
\hline \multicolumn{11}{|c|}{ Missing teeth } \\
\hline No & $4(20.0)$ & $13(65.0)$ & $3(15.0)$ & 20 (100.0) & 0.916 & $4(22.2)$ & $11(61.1)$ & $3(16.7)$ & 18 (100.0) & 0.787 \\
\hline Yes & 20 (16.3) & $83(67.4)$ & 20 (16.3) & 123 (100.0) & & $20(16.0)$ & 85 (68.0) & $20(16.0)$ & 125 (100.0) & \\
\hline \multicolumn{11}{|c|}{ Incidence of missing teeth } \\
\hline No & - & - & - & - & - & $20(21.8)$ & $59(64.1)$ & $13(14.1)$ & $92(100.0)$ & 0.094 \\
\hline Yes & - & - & - & - & - & $4(7.8)$ & $37(72.6)$ & $10(10.6)$ & $51(100.0)$ & \\
\hline \multicolumn{11}{|l|}{ Bleeding } \\
\hline No & $12(19.0)$ & $43(68.3)$ & $08(12.7)$ & $63(100.0)$ & 0.558 & $11(12.8)$ & $60(69.8)$ & $15(17.4)$ & $86(100.0)$ & 0.284 \\
\hline Yes & $12(15.0)$ & $53(66.2)$ & 15 (18.8) & $80(100.0)$ & & $13(22.8)$ & $36(63.2)$ & $8(14.0)$ & $57(100.0)$ & \\
\hline \multicolumn{11}{|c|}{ Periodontal pockets } \\
\hline No & $22(22.0)$ & $69(69.0)$ & $9(9.0)$ & $100(100.0)$ & $<0.001$ & $21(22.6)$ & $62(66.7)$ & 10 (10.7) & $93(100.0)$ & 0.007 \\
\hline Yes & $2(4.6)$ & $27(62.8)$ & $14(32.6)$ & $43(100.0)$ & & $3(6.0)$ & $34(68.0)$ & $13(26.0)$ & $50(100.0)$ & \\
\hline \multicolumn{11}{|c|}{ Oral health related quality of life } \\
\hline No & $14(19.4)$ & $49(68.1)$ & $9(12.5)$ & $72(100.0)$ & 0.409 & $16(19.0)$ & $58(69.1)$ & $10(11.9)$ & $84(100.0)$ & 0.229 \\
\hline Yes & $10(14.1)$ & $47(66.2)$ & 14 (19.7) & $71(100.0)$ & & $8(13.6)$ & $38(64.4)$ & $13(22.0)$ & $59(100.0)$ & \\
\hline
\end{tabular}

Some variables do not add $n=143$ for containing missing data. ${ }^{*}$ Data referring to the collection held in 2011 .

professional dental practice and public health efforts to prevent these diseases, these factors are still among the most prevalent in the world, and no reduction in their prevalence has been observed in adults in the last decades. ${ }^{4,6}$ This situation was also observed in this study, where the results for occurrence of caries and periodontal disease did not decrease in the fouryear study period.

In this study, tooth decay was associated with variables of inequalities in 2011, with higher prevalence in the lowest social class level, and with public dental service users. However, in the 2015 follow-up, this association was not significant, thereby showing a reduction in the impact of inequalities on caries. This may reflect the investment in the public dental service in the country, by implementation of the National Oral Health Policy in 2004, to meet the aim of integrating the country's healthcare into SUS. SUS is now the largest public health system in the world, and one of its principles is equity. In the last 15 years, coverage of the Family Health Strategy (FHS) has extended its access to primary healthcare, especially in the country's poorest regions, and has improved its access to health services among vulnerable populations ${ }^{10,22}$. Despite the world economic crisis, the authors observed an increase in access to healthcare in Brazil between 2008 and 2013, including the FHS, and both medical 
Reducing social inequalities in the oral health of an adult population

Table 3. Association between type of service used and adult oral health variables. Piracicaba, SP, Brazil, $2011-2015$.

\begin{tabular}{|c|c|c|c|c|c|c|c|c|c|c|}
\hline \multirow{4}{*}{$\begin{array}{l}\text { Oral health } \\
\text { variables }\end{array}$} & \multicolumn{10}{|c|}{ Type of service } \\
\hline & \multicolumn{5}{|c|}{ Baseline } & \multicolumn{5}{|c|}{ Follow-up } \\
\hline & Private & Insurance & Public & \multirow{2}{*}{ Total } & \multirow{2}{*}{$p$-value } & Private & Insurance & Public & \multirow{2}{*}{ Total } & \multirow{2}{*}{$p$-value } \\
\hline & n (\%) & n (\%) & n (\%) & & & n (\%) & n (\%) & n (\%) & & \\
\hline \multicolumn{11}{|c|}{ Visible biofilm } \\
\hline No & $41(50.0)$ & $26(31.7)$ & 15 (18.3) & $82(100.0)$ & 0.130 & 47 (55.3) & $24(28.2)$ & $14(16.5)$ & 85 (100.0) & 0.013 \\
\hline Yes & $23(42.6)$ & $13(24.1)$ & $18(33.3)$ & 54 (100.0) & & $46(50.0)$ & $14(26.4)$ & $20(37.7)$ & 53 (100.0) & \\
\hline \multicolumn{11}{|c|}{ Decayed teeth } \\
\hline No & $46(48.9)$ & $32(34.1)$ & $16(17.0)$ & $94(100.0)$ & 0.010 & $21(42.8)$ & $26(28.3)$ & $20(21.7)$ & 92 (100.0) & 0.618 \\
\hline Yes & 21 (44.7) & $08(17.0)$ & 18 (38.3) & 47 (100.0) & & & $14(28.6)$ & $14(28.6)$ & 49 (100.0) & \\
\hline \multicolumn{11}{|l|}{ Filled teeth } \\
\hline No & 09 (45.0) & 03 (15.0) & 08 (40.0) & 20 (100.0) & 0.140 & 09 (53.0) & 04 (23.5) & $04(23.5)$ & 17 (100.0) & 0.869 \\
\hline Yes & $58(47.9)$ & 37 (30.6) & $26(21.5)$ & 121 (100.0) & & $58(46.8)$ & $36(29.0)$ & $30(24.2)$ & $124(100.0)$ & \\
\hline \multicolumn{11}{|l|}{ Missing teeth } \\
\hline No & 07 (36.8) & 06 (31.6) & 06 (31.6) & 19 (100.0) & 0.573 & $05(29.4)$ & 06 (35.3) & 06 (35.3) & 17 (100.0) & 0.264 \\
\hline Yes & 60 (49.2) & 34 (27.9) & 28 (22.9) & 122 (100.0) & & $62(50.0)$ & $34(27.4)$ & $28(22.6)$ & 124 (100.0) & \\
\hline \multicolumn{11}{|c|}{ Incidence of missing teeth } \\
\hline No & - & - & - & - & - & $40(44.4)$ & $32(35.6)$ & $18(20.0)$ & $90(100.0)$ & 0.034 \\
\hline Yes & - & - & - & - & - & $27(52.9)$ & $08(15.7)$ & $16(31.4)$ & $51(100.0)$ & \\
\hline \multicolumn{11}{|l|}{ Bleeding } \\
\hline No & $31(80.8)$ & $16(26.2)$ & $14(23.0)$ & $61(100.0)$ & 0.786 & $43(51.2)$ & $25(29.8)$ & $16(19.0)$ & $84(100.0)$ & 0.230 \\
\hline Yes & $36(45.0)$ & $24(30.0)$ & $20(25.0)$ & $80(100.0)$ & & $24(42.1)$ & $15(26.3)$ & $18(31.6)$ & $57(100.0)$ & \\
\hline \multicolumn{11}{|c|}{ Periodontal pockets } \\
\hline No & $49(50.0)$ & $29(29.6)$ & $20(20.4)$ & $98(100.0)$ & 0.299 & $49(53.8)$ & $23(25.3)$ & $19(20.9)$ & $91(100.0)$ & 0.126 \\
\hline Yes & $18(41.9)$ & $11(25.6)$ & $14(32.5)$ & $43(100.0)$ & & $18(36.0)$ & $17(34.0)$ & $15(30.0)$ & $50(100.0)$ & \\
\hline \multicolumn{11}{|c|}{ Oral health related quality of life } \\
\hline No & $29(41.4)$ & $27(38.6)$ & $14(20.0)$ & 70 (100.0) & 0.028 & $42(50.6)$ & $23(27.7)$ & $18(21.7)$ & $83(100.0)$ & 0.629 \\
\hline Yes & 38 (53.5) & 13 (18.3) & $20(28.2)$ & 71 (100.0) & & $25(43.1)$ & 17 (29.3) & $16(27.6)$ & 58 (100.0) & \\
\hline
\end{tabular}

Some variables do not add $n=143$ for containing missing data.

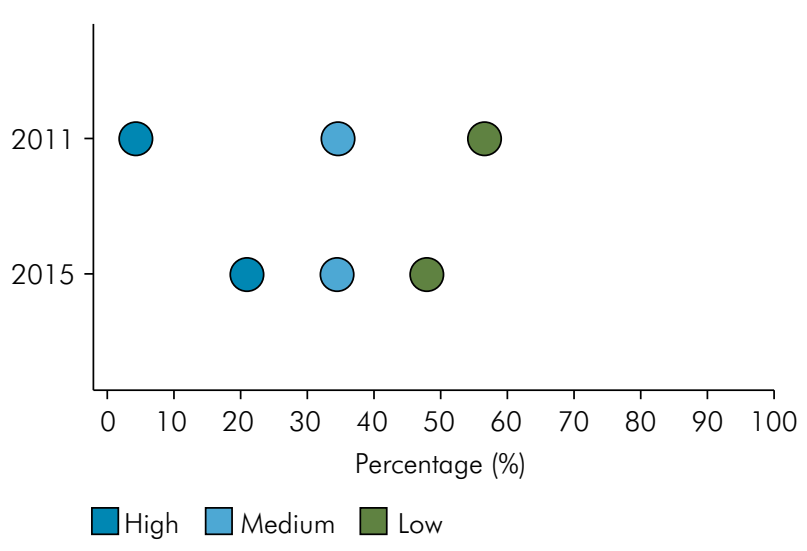

Figure 1. Reduction in inequality of dental caries prevalence according to social class, among adults residing in Piracicaba, SP, Brazil, $2011-2015$. and dental visits ${ }^{23}$. We also observed a smaller disparity between rich and poor users of the FHS or health insurance. ${ }^{23}$ Moreover, Brazil has undergone substantial socioeconomic changes in the last decades, which have reduced poverty and improved living conditions. ${ }^{24}$ Considering Brazil's current scenario of political and financial crisis, it goes without saying that the investments in social and health policies should be maintained; otherwise, all gains achieved in the country thus far could be lost. ${ }^{23}$

Even though Brazil has improved considerably in addressing the suppressed demands of its population, more must be done if it ever expects to reduce the occurrence of preventable oral diseases. In regard 

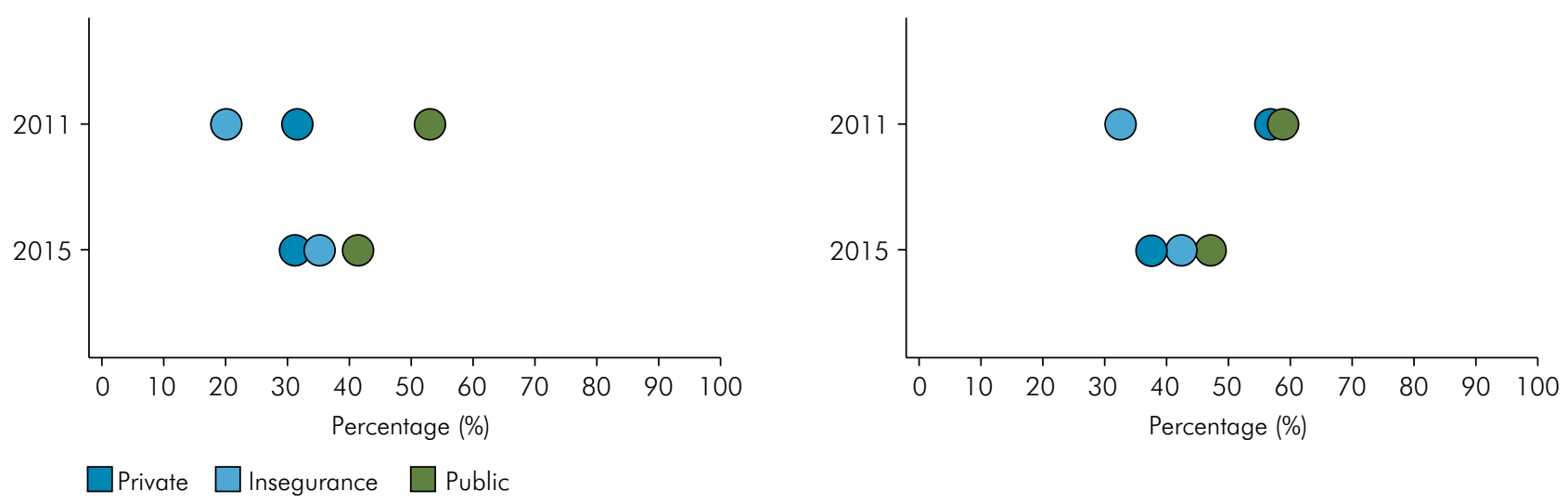

Figure 2. Reduction in (A) inequality of dental caries prevalence, and (B) impact on oral health-related quality of life, according to type of dental service used, among adults residing in Piracicaba, SP, Brazil, 201 1-2015.

to periodontal disease, the lowest social class was associated with the presence of periodontal pockets, a result that remained unchanged during the study. Some studies have shown stronger association between periodontal diseases and socioeconomic factors. ${ }^{25}$ Ecological studies found that the highest levels of income inequality in developed countries, according to the Gini coefficient, were significantly associated with periodontal disease severity in adults. ${ }^{26}$ A possible explanation would be the lack of both regular clinical visits and clinical follow-up, resulting from inadequate access to dental services. In addition, some studies emphasize greater association of periodontal disease with socioeconomic, psychosocial and behavioral factors and stress, ${ }^{25,27}$ in comparison with other oral health conditions, such as tooth decay and oral cancer. ${ }^{28}$

Visible biofilm is an essential predisposing factor for the occurrence of caries and periodontal disease; therefore, measures should be put in place to reduce it by ensuring the population's oral health. Although our study found a reduction in the impact of inequalities on tooth decay-but not on periodontal disease-it observed an alarming high prevalence of visible biofilm in the population studied. More importantly, this condition remained unchanged between 2011 and 2015 regarding social class, and was associated with the type of public service in 2015. In this respect, it is hard to imagine how reducing the impact on oral diseases, tooth loss and quality of life of most of the sample can be minimized if no measures are put into place to prevent or reduce the incidence of these diseases. In this regard, health services should promote the autonomy and co-responsibility of users regarding their health condition, so that healthier choices may be made, and better health behaviors may be adopted, a partnership that can be established through primary healthcare.

The literature addresses an association between negative perception of oral health among lower income individuals and less education. This reflects a worsening of oral health conditions and social inequalities..$^{29,30}$ Nevertheless, the impact of oral health on quality of life, observed in this study, was associated only with the type of dental service used at baseline. In addition, the impact on quality of life was the only oral health condition that had a significant reduction in prevalence in 2015 versus 2011. Although negative self-perception was reduced in 2015, the authors observed that disease prevalence did not fall in the study population; therefore, more value should be ascribed to improving the means of promoting self-perception and seeking health services.

The study limitations included sample loss, mainly in the age group studied, the follow-up time and the population-based sample. Since adults are economically active, they are hard to find in households, and the information about them is difficult to keep track of due to home address change. However, the socioeconomic characteristics of the study profile for the representative sample at the study 
location were maintained from baseline to follow-up. ${ }^{19}$ This may lessen the chance of sample selection bias, further reduced by allowing only individuals who participated in both phases of the investigation to be studied, to ensure a valid comparison between the two study periods.

Our findings revealed that there is still a high prevalence of oral diseases in the adult population, including biofilm as a predisposing factor, tooth decay, periodontal pockets and its sequelae, tooth loss and quality of life. Other studies have associated the greater occurrence of oral diseases with individuals of lower income gradients, educational level and social class. ${ }^{25,26}$ There is evidence to support the association between oral disease and living conditions of individuals. It is known that inadequate individual behavior is influenced by the social context; therefore, it follows that poor health is associated with SDH. ${ }^{31}$ Social determinants can affect oral health conditions, leading to greater exposure to risk factors, and hindering access to and use of oral health services. Hence, efforts must be made to carry out integrated interventions for the prevention and promotion of oral health. ${ }^{32}$

Health promotion strategies should encompass all determinants ${ }^{33}$, ranging from the improvement of the population's socioeconomic conditions to greater control over risk factors. Fair public policies can be expected to achieve positive outcomes that include creating economic opportunities to encourage the building of support networks and increase the ability of individuals to address local and global issues, fostering their relations with other groups, strengthening their organization and participation in collective actions, and committing to their role of social players and active participants in social life decisions. ${ }^{9}$ On the other hand, public health interventions introduced without any strategic planning may produce an undesirable effect and widen health inequalities, instead of promoting the universalization of benefits or allowing additional direct funds to be granted to groups with greater needs. This effect is called inverse equity, that is to say, groups with better socioeconomic conditions absorb the benefits of public policies earlier and more intensely. This increases inequalities in health. In the future, a reduction in oral diseases and their physical and social sequelae can be expected to preserve teeth better, which is a determining factor in reducing the impact of oral diseases on the quality of life of individuals. ${ }^{30}$

Finding a solution to the problem of health inequalities remains a challenge, ${ }^{32}$ but the adoption of strategies to reduce the disease burden and improve access to healthcare is fundamental to achieving better results for these health disparities. A model policy must be adopted in all countries to reduce the burden of diseases in an equitable manner. Although the Unified Health System is recent in Brazil, specifically the National Oral Health Policy, it has already minimized the polarization of oral diseases, mostly affecting the lower socioeconomic strata. Moreover, it is expected to continue impacting other oral health conditions, such as dental loss, in the long-term. It is noteworthy that this adult population has experienced the benefits of a very exclusive health system, especially regarding oral healthcare. An increase in FHS coverage is expected to positively impact the continuous care of the population in the long-term.

\section{Conclusion}

No reduction was found in the prevalence of oral health conditions, and an association was observed among the lowest social class, use of public services and worse oral health conditions (presence of visible biofilm, tooth decay, periodontal pockets and oral health impact on self-perceived quality of life). Nevertheless, there was a reduction in inequalities regarding dental caries, and in the impact on the oral health-related quality of life of adults belonging to the lower social class, in the study period.

These results suggest that, although the Brazilian National Oral Health Policy is recent, it has achieved its mission, especially that of integrality and equity. In this regard, the scope and reach of the Brazilian Unified Health System should be duly valued, and should serve as a model for other countries to implement their own universal healthcare system. 


\section{Acknowledgements}

The authors would like to thank Professor Antonio Carlos Frias for contributing to the study design, and Fundação de Amparo à Pesquisa do Estado de São Paulo - FAPESP - (2009/16560-0 and 2011/00545-1) for supporting this study through its Cooperation Agreement, and Coordenação de Aperfeiçoamento de Pessoal de Nível Superior - CAPES (2014/15184-2). We also wish to thank Espaço da Escrita - Coordenadoria Geral da Universidade - UNICAMP - for its translation services.

\section{References}

1. Arcaya MC, Arcaya AL, Subramanian SV. Inequalities in health: definitions, concepts, and theories. Glob Health Action. 2015 Jun;8(27106):27106. https://doi.org/10.3402/gha.v8.27106

2. Frenk J, Moon S. Governance challenges in global health. N Engl J Med. 2013 Mar;368(10):936-42. https://doi.org/10.1056/NEJMral109339

3. Thomson WM. Social inequality in oral health. Community Dent Oral Epidemiol. 2012 Oct;40 Suppl 2:28-32. https://doi.org/10.1111/j.1600-0528.2012.00716.x

4. Kassebaum NJ, Bernabé E, Dahiya M, Bhandari B, Murray CJ, Marcenes W. Global burden of untreated caries: a systematic review and metaregression. J Dent Res. 2015 May;94(5):650-8. https://doi.org/10.1177/0022034515573272

5. Kassebaum NJ, Bernabé E, Dahiya M, Bhandari B, Murray CJ, Marcenes W. Global burden of severe tooth loss: A systematic review and meta-analysis. J Dent Res. 2014 Jul;93(7 Suppl):20S-8S. https://doi.org/10.1177/0022034514537828

6. Kassebaum NJ, Bernabé E, Dahiya M, Bhandari B, Murray CJ, Marcenes W. Global burden of severe periodontitis in 1990-2010: a systematic review and meta-regression. J Dent Res. 2014 Nov;93(11):1045-53. https://doi.org/10.1177/0022034514552491

7. Silva-Junior MF, Sousa AC, Batista MJ, Sousa MD. [Oral health condition and reasons for tooth extraction among an adult population (20-64 years old)]. Cien Saude Colet. 2017 Aug;22(8):2693-702. Portuguese. https://doi.org/10.1590/1413-81232017228.22212015

8. Marcenes W, Kassebaum NJ, Bernabé E, Flaxman A, Naghavi M, Lopez A, et al. Global burden of oral conditions in 1990-2010: a systematic analysis. J Dent Res. 2013 Jul;92(7):592-7. https://doi.org/10.1177/0022034513490168

9. Buss PM, Pellegrini Filho A. Iniqüidades em saúde no Brasil, nossa mais grave doença: comentários sobre o documento de referência e os trabalhos da Comissão Nacional sobre Determinantes Sociais da Saúde. Cad Saude Publica. 2006 Sep;22(9):2005-8. https://doi.org/10.1590/S0102-311X2006000900033

10. Pucca Junior GA, Gabriel M, Araujo ME, Almeida FC. Ten years of a national oral health policy in Brazil: innovation, boldness, and numerous challenges. J Dent Res. 2015 Oct;94(10):1333-7. https://doi.org/10.1177/0022034515599979

11. Peres KG, Peres MA, Boing AF, Bertoldi AD, Bastos JL, Barros AJ. Reduction of social inequalities in utilization of dental care in Brazil from 1998 to 2008. Rev Saude Publica. 2012 Apr;46(2):250-8. https://doi.org/10.1590/S0034-89102012000200007

12. Monteiro CN, Beenackers MA, Goldbaum M, Barros MBA, Gianini RJ, Cesar CL, et al. Socioeconomic inequalities in dental health services in Sao Paulo, Brazil, 2003-2008. BMC Health Serv Res. 2016 Dec;16(1):683. https://doi.org/10.1186/s12913-016-1928-y

13. Jung SH, Kim MH, Ryu Jl. Inequalities in oral health among adolescents in Gangneung, South Korea. BMC Oral Health. 2018 Apr; 18(1):68. https://doi.org/10.1186/s12903-018-0533-3

14. Murakami K, Ohkubo T, Nakamura M, Ninomiya T, Ojima T, Shirai K, et al. Socioeconomic inequalities in oral health among middle-aged and elderly Japanese: NIPPON DATA 2010. J Epidemiol. 2018;28 Suppl 3:S59-65. https://doi.org/10.2188/jea.JE20170247

15. Singh A, Harford J, Antunes JL, Peres MA. Area-level income inequality and oral health among Australian adults-A population-based multilevel study. PLoS One. 2018 Jan;13(1):e0191438. https://doi.org/10.1371/journal.pone.0191438

16. Moradi G, Moinafshar A, Adabi H, Sharafi M, Mostafavi F, Bolbanabad AM. Socioeconomic inequalities in the oral health of people aged 15-40 years in Kurdistan, Iran in 2015: a cross-sectional study. J Prev Med Public Health. 2017 Sep;50(5):303-10. https://doi.org/10.3961/ipmph.17.035

17. Prado RL, Garbin CA, Moimaz SA, Saliba NA. Inequalities in oral health among older Brazilians: a cross-sectional analysis of a National survey. Pesq Bras Odontoped Clin Integr. 2018;18(1):e3726. https://doi.org/10.4034/PBOCI.2018.181.12.

18. Guarnizo-Herreño CC, Watt RG, Garzón-Orjuela N, Tsakos G. Explaining oral health inequalities in European welfare state regimes: the role of health behaviours. Community Dent Oral Epidemiol. 2019 Feb;47(1):40-8. https://doi.org/10.1111/cdoe.12420

19. Silva-Junior MF, Sousa MD, Batista MJ. Prospective cohort of adult oral health in Piracicaba, SP, Brazil. BMC Res Notes. 2019 Apr;12(1):221. https://doi.org/10.1186/s13104-019-4243-y

20. Graciano MI, Lehfeld NA, Neves Filho A. Critérios de avaliação para classificação socioeconômica: elementos de atualização. Serv Soc Real. 1999;8(1):109-28.

21. Oliveira BH, Nadanovsky P. Psychometric properties of the Brazilian version of the Oral Health Impact Profile-short form. Community Dent Oral Epidemiol. 2005 Aug;33(4):307-14. https://doi.org/10.1111/i.1600-0528.2005.00225.x 
Reducing social inequalities in the oral health of an adult population

22. Victora CG, Barreto ML, Leal MC, Monteiro CA, Schmidt MI, Paim J, et al. Health conditions and health-policy innovations in Brazil: the way forward. Lancet. 2011 Jun;377(9782):2042-53. https://doi.org/10.1016/S0140-6736(11)60055-X

23. Mullachery P, Silver D, Macinko J. Changes in health care inequity in Brazil between 2008 and 2013. Int J Equity Health. 2016 Nov;15(1):140. https://doi.org/10.1186/s12939-016-0431-8

24. Paim J, Travassos C, Almeida C, Bahia L, Macinko J. The Brazilian health system: history, advances, and challenges. Lancet. 2011 May;377(9779):1778-97. https://doi.org/10.1016/S0140-6736(11)60054-8

25. Sheiham A, Nicolau B. Evaluation of social and psychological factors in periodontal disease. Periodontol 2000. 2005;39(1):118-31. https://doi.org/10.1111/j.1600-0757.2005.00115.x

26. Sabbah W, Sheiham A, Bernabé E. Income inequality and periodontal diseases in rich countries: an ecological cross-sectional study. Int Dent J. 2010 Oct;60(5):370-4.

27. Sabbah W, Tsakos G, Sheiham A, Watt RG. The role of health-related behaviors in the socioeconomic disparities in oral health. Soc Sci Med. 2009 Jan;68(2):298-303. https://doi.org/10.1016/i.socscimed.2008.10.030

28. Hobdell MH, Oliveira ER, Bautista R, Myburgh NG, Lalloo R, Narendran S, et al. Oral diseases and socio-economic status (SES). Br Dent J. 2003 Jan;194(2):91-6. https://doi.org/10.1038/sj.bdj.4809882

29. Batista MJ, Lawrence HP, Sousa ML. Impact of tooth loss related to number and position on oral health quality of life among adults. Health Qual Life Outcomes. 2014 Nov;12(1):165. https://doi.org/10.1186/s12955-014-0165-5

30. Luchi CA, Peres KG, Bastos JL, Peres MA. Inequalities in self-rated oral health in adults. Rev Saude Publica. 2013 Aug;47(4):740-51. https://doi.org/10.1590/S0034-8910.2013047004364

31. Strategic Review of Health Inequalities in England Post-2010. Fair society, healthy lives: the marmot review. [S. I.]: Marmot Review; 2010 [cited 2015 Jun 19]. 2010. Available from: http://www.instituteofhealthequity.org/resources-reports/fair-society-healthy-lives-the-marmot-review/

32. Marmot M, Bell R. Social determinants and dental health. Adv Dent Res. 2011 May;23(2):201-6. https://doi.org/10.1177/0022034511402079

33. Sheiham A, Alexander D, Cohen L, Marinho V, Moysés S, Petersen PE, et al. Global oral health inequalities: task group-implementation and delivery of oral health strategies. Adv Dent Res. 2011 May;23(2):259-67. https://doi.org/10.1177/0022034511402084 